BMJ Open Sport \& Exercise Medicine

\title{
Effects of one week different intensity endurance exercise on cardiorespiratory and cardiometabolic markers in junior young athletes
}

\author{
Gashaw Tesema, ${ }^{\oplus 1,2}$ Mala George, ${ }^{3}$ Soumitra Mondal, ${ }^{4}$ D Mathivana ${ }^{4}$
}

\begin{abstract}
To cite: Tesema G, George M, Mondal S, et al. Effects of one week different intensity endurance exercise on cardiorespiratory and cardiometabolic markers in junior young athletes. BMJ Open Sport \& Exercise Medicine 2019;5:e000644. doi:10.1136/ bmjsem-2019-000644
\end{abstract}

Accepted 10 September 2019

Check for updates

(C) Author(s) (or their employer(s)) 2019. Re-use permitted under CC BY. Published by BMJ.

${ }^{1}$ Department of Sport Science, College of Natural and Computational science, MizanTepi University, Tepi, Ethiopia ${ }^{2}$ Department of Sport Science, College of Natural and Computational Sciences, Mekelle University, Mekelle, Ethiopia

${ }^{3}$ Department of Biochemistry, College of Health Sciences, Mekelle University, Mekelle, , Ethiopia

${ }^{4}$ Department of Sport science, College of Natural and Computational Sciences, Mekelle University, Mekelle, , Ethiopia

\section{Correspondence to}

Dr Gashaw Tesema, MizanTepi University, Mizzan 1933, Ethiopia;

gashtesema@gmail.com

\section{ABSTRACT}

Background Endurance training in varied intensity zones are more likely to show different adaptive responses. However, it is still unclear the intensity level of endurance exercise in junior young athletes that may promote increased markers for cardiorespiratory and cardiometabolic response. Therefore, we aimed to investigate the effect of 1 week different intensity endurance training on markers of cardiorespiratory and cardiometabolic response in young athletes.

Methods Thirty apparently healthy male young athletes averaging $19.4 \pm 1.3$ years of age were recruited and randomly assigned to two different intensity endurance training groups. The first group trained at $60 \%$ to $70 \%$ maximum heart rate (HRmax) for 45 min per session and the second group trained at $70 \%$ to $80 \%$ HRmax for $30 \mathrm{~min}$ per session for a period of 1 week three sessions per week. A pre-training and post-training, marker of cardiorespiratory and cardiometabolic response was assessed for both groups.

Results Both intensity training had no significant effect on markers of cardiorespiratory response within and between training groups $(p>0.05)$. Whereas, cardiometabolic markers lactate dehydrogenase $(p=0.001)$, cardiac troponin I $(p=0.001)$ and creatinekinase myocardial band $(\mathrm{p}=0.001)$ significantly increased at the end of 1 week within the group in both training groups $(p \leq 0.05)$. In the comparative analysis between groups, there was no significant difference in markers of cardiometabolic response $(p>0.05)$.

Conclusion 1 week $60 \%$ to $70 \%$ HRmax and $70 \%$ to $80 \%$ HRmax intensity zones induces similar acute effects on markers for cardiometabolic and cardiorespiratory responses between groups and significant elevations of cardiometabolic markers within the groups in moderately trained young athletes.

\section{INTRODUCTION}

Endurance events are becoming increasingly popular ${ }^{1}$ both in young and recreational athletes. Although regular physical activity exerts a range of beneficial physiological effects on cardiovascular health, but a long-duration ultra-endurance exercise might produce substantial changes in

\section{Key messages}

What are the new findings

- Different endurance training intensity zones $(60 \%$ to $70 \%$ maximum heart rate (HRmax) and $70 \%$ to $80 \%$ HRmax) responses similar acute effects on cardiometabolic and cardiorespiratory markers.

- Elevations of serum cardiometabolic markers have been observed similarly on both intensity zones in young athletes.

\section{How might it impact on clinical practice in the} near future

- Evaluation of cardiometabolic markers of athletes during clinical evaluation might be false-positive which need careful assessment using different measurement evaluations.

cardiometabolic parameters ${ }^{2}{ }^{3}$ and muscle damage. ${ }^{4}$ The research published so far reported several result differences for many reasons, such as the variation in the duration and intensity of the physical exercises. ${ }^{5}$ In addition, studies involving intensifications of training in already well-trained athletes have shown equivocal results. ${ }^{5}{ }^{6}$ However, little information is available on the effects of different intensities, in the modulation of the markers for cardiorespiratory and cardiometabolic stress especially in young moderately trained athletes. Therefore, such knowledge could help young and recreational athletes create effective exercise programmes for muscle strength and cardiorespiratory adaptation.

The optimum training intensity for eliciting maximum performance adaptation in endurance exercise at steady state remains inconclusive. Effect of high intensity exercise on skeletal and cardiac muscle cells results into increased metabolic stress markers. ${ }^{78}$ Thus, higher values than normal serum cardiac stress markers like cardiac troponin I (cTnI) ${ }^{9} 10$ creatinekinase myocardial band (CK-MB) and lactate dehydrogenase (LDH),${ }^{11} 12$ have been 
reported. Interestingly, a study by Peake $e t a l^{13}$ found out that, there were significant elevation in serum cardiac markers in response to both moderate and high intensity training. However, studies by Daussin et al, Helgerud $e t$ $a l$ and Gorostiaga et $a l,{ }^{14-16}$ reported no difference in the increase in maximum oxygen consumption $\left(\mathrm{VO}_{2} \max \right)$ between high and moderate intensity exercise training. Therefore, the above inconclusive findings could be due to either smaller or bigger differences in exercise intensities. Hence, endurance training in varied intensity zones, are more likely to show different adaptive responses.

It is still unclear the intensity level of endurance exercise that may promote increased markers for cardiorespiratory and cardiometabolic after short-term exposure to endurance exercise training in moderately trained athletes. Therefore, in this study, we aimed to observe the immediate effect at recovery after 1 week different intensity endurance training on cardiorespiratory and cardiometabolic markers of young athletes. Hence, we applied 1 week endurance training $60 \%$ to $70 \%$ maximum heart rate (HRmax) for 45 min per session for the first group and $70 \%$ to $80 \%$ HRmax for 30 min per session for a period of 1 week for the second group for 3days per week $\mathrm{k}^{-1}$. The duration was as it is recommended for improving and maintaining cardiorespiratory fitness for health purposes. ${ }^{17}$

\section{METHODS}

\section{Study area}

This study was conducted in Bahir Dar, located about $578 \mathrm{~km}$ north-northwest of Addis Ababa, Ethiopia. It has an altitude of 1840 metres above the sea level and within latitude and longitude of $11^{\circ} 36^{\prime} \mathrm{N} 37^{\circ} 23^{\prime} \mathrm{E}$ coordinates, while the annual average temperature is $25^{\circ} \mathrm{C}$ to $32^{\circ} \mathrm{C}$, with an average humidity of $58 \%$. $^{18}$

\section{Participants and sample size}

Thirty apparently healthy male young athletes within $19.4 \pm 1.3$ years of age were recruited from Bahir Dar University Sport Academy. All participants were moderately trained and active in involving in ball games and athletics trainings given in the sport academy and familiar to a workload of 3 days per week.

The sample size was based on similar studies done before ${ }^{19}$ using the following equation ${ }^{20}$ which considers $80 \%$ power and $\mathrm{p} \leq 0.05$. Where, $\mathrm{n}=$ sample size needed per group, $\sigma^{* 2}$ T is variance of the treatment, $\sigma^{* 2} R$ is variance of the reference, $\delta$ is the maximum percent of no clinical importance, CVT is coefficient of variations of the treatment, CVR is coefficient of variations of the reference.

$$
n=\frac{(z \alpha+z \beta / 2)^{2}(\sigma 2+\sigma 2) R * T *}{(\delta-/ C V T-C V R /)^{2}}, \quad n=\frac{(1.64+0.84)^{2}\left(0.30^{2}+0.35^{2}\right)}{(0.10+0.70-0.50)^{2}}=14.5
$$

Consequently, a validated Physical Activity Readiness Questionnaire $^{21}$ was used to evaluate conditions that may prohibit participants from practising in endurance training.

\section{Inclusion and exclusion criteria}

Participants that reported problems in their health (heart dysfunction and chest pain during exercise) and physical conditions (loss of balance, consciousness and bone or joint problem) and are currently taking antihypertensive drugs were excluded from the study. In addition, smokers and alcoholics were also excluded from participating in the study due to reported influences on both metabolic and physical performance biomarkers. ${ }^{22}{ }^{23}$ Participants with age 18 to 25 years of old were included. This is due to the reason that fitness of sports men above 25 years becomes decline and fitness of youngsters with age below 18 years is in the process of growing which can be confounding factors for the result. Only male athletes also included in the study for maintaining homogeneity of the experiment, moderately trained athletes, who fulfils the Physical Activity Readiness Questionnaires and passed the aforementioned exclusion criteria were included in the study.

\section{Study design}

A randomised, parallel group study design was used in this study. Randomisation to endurance exercise is illustrated in (figure 1). Thus, 15 each of the 30 subjects participating in this study, was randomly assigned to two different intensity endurance exercise group. Pre-training and post-training, cardiorespiratory as well as cardiometabolic markers were assessed for both groups. Finally, post training within the group and between groups comparison was done.

\section{Training protocol and ethical approval}

This field based study conducted based on the protocol specified here. Two professional athletics coaches are assigned one for the first and one for the second training groups. A 5 min warming up and stretching exercise given at the beginning of the training and $5 \mathrm{~min}$ of cooling down were also given at the end of each training time for both groups. Then continuous endurance running was given for $45 \mathrm{~min}$ at $60 \%$ to $70 \% \mathrm{HRmax}$ for the first intensity training group and $30 \mathrm{~min}$ at $70 \%$ to $80 \% \mathrm{HRmax}$ for the second intensity training group in the open field track per session for 3 days per week and it continued for 1 week. The training intensity was monitored with polar heart rate monitor throughout the entire session. The heart rate monitor adjusted to the designed percent maximum heart rate for both groups guided us whether it is bellow or above the designed \% HRmax to adjust the heart rate drift throughout the training. We aimed to observe acute effects of the training on cardiometabolic markers as we are not expecting to get effective changes on cardiorespiratory markers. Both groups were instructed not to participate in other exercise training and to continue the usual habit of nutrition throughout the experiment.

Ethical approval was obtained from Institutional Research Ethics Review Committee of Mekelle University conformed to the 1975 Declaration of Helsinki. Written 


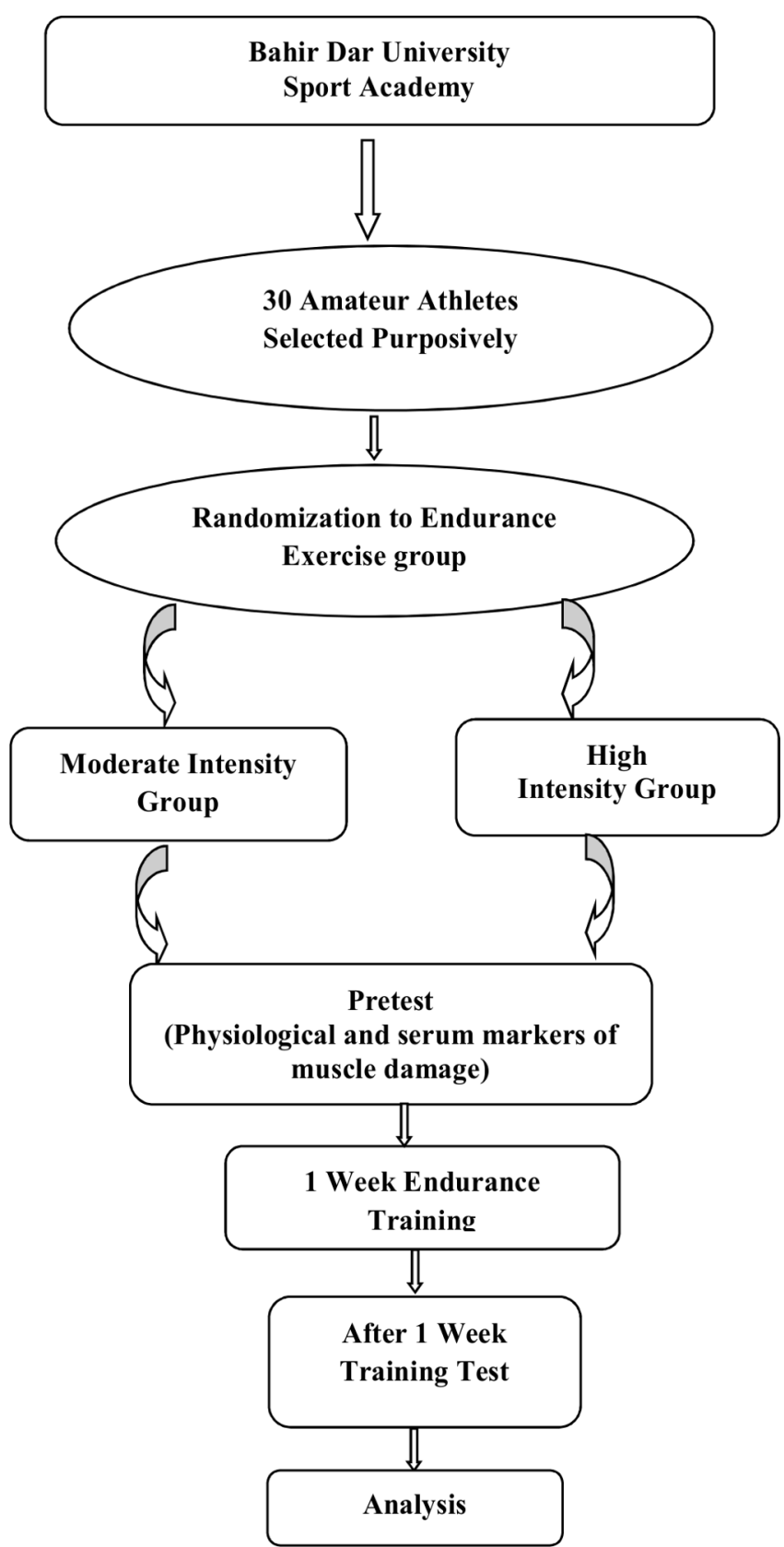

Figure 1 Flow chart for study design and data collection.

consent was delivered to the participants and they were informed about the objectives of the study. Participation in this study was purely a voluntary activity and their right not to participate was respected. Issues of confidentiality and anonymity were maintained too.

\section{Measurements of cardiorespiratory markers}

Cardiorespiratory markers $\left(\mathrm{VO}_{2} \max\right.$, blood pressure and resting heart rate) were assessed just before beginning (pre-test) and after 1 week post-training test. $\mathrm{VO}_{2} \max$ of subjects was estimated using 3 min step test. Subjects stepped up and down a 16.5 inch stepping bench with a rate of 96 steps per minutes for $3 \mathrm{~min}$ in time of metronome beep sound. Five seconds after exactly $3 \mathrm{~min}$ pulse was taken from radial artery with heart rate monitor tied around the wrist. Finally maximal aerobic power $\left(\mathrm{VO}_{2} \max \right)$ was calculated using the equation $\mathrm{VO}_{2} \max =111.33-(0.42 \times$ maximum pulse measured $5 \mathrm{~s}$ after stepping for $3 \mathrm{~min})^{24}$ and reported in $(\mathrm{ml} / \mathrm{kg} / \mathrm{min})$.

Systolic blood pressure (SBP) and diastolic blood pressure (DBP) was measured with Spengler digital electronic sphygmomanometer. The tourniquet of sphygmomanometer was tied on the wrist of the lower arm and inflated with air while subject seated. The result displayed from the indicator of sphygmomanometer was recorded in millimetre mercury. Mean arterial pressure (MAP) was computed using the following formula: $\mathrm{MAP}=\mathrm{DBP}+1 / 3$ (SBP-DBP). ${ }^{25}$

Measurement of resting heart rate was done in the academy dormitory in the morning immediately after wake up using a heart rate monitor. The heart rate monitor sensor was tied on the radial artery of the hand and the subject laid down relaxed on supine position. After about $1 \mathrm{~min}$, the heart rate monitor was started. The subject breathed calmly for 3 to $5 \mathrm{~min}$ without looking at the heart rate monitor. Then after 3 to $5 \mathrm{~min}$ the heart rate monitor was stopped. Finally the result of heart rate was checked and recorded.

\section{Assays of serum cardiometabolic markers}

Blood samples of $5 \mathrm{~mL}$ were collected just before training (pre-test) from each participant. Then, post-training samples collected 4 hours after the final training session at the end of 1 week endurance training since the peak cardiometabolic markers are achieved 3 to 4 hours after training time. ${ }^{2627}$ Blood was taken from an antecubital vein using Vacutainer Serum Separator Tube (SST) containing blood clotting accelerant gel. The serum was separated by centrifugation of the blood sample at $4000 \mathrm{rpm}$ (revolution per minute) for $3 \mathrm{~min}$ and stored at $-20^{\circ} \mathrm{C}$ until analysis. The levels of $\mathrm{LDH}$ were measured using a spectrophotometric assay with BS-2E chemistry analyser according to the guidelines of the International Federation of Clinical Chemistry (Beckman Coulter, Krefeld, Germany). While, the levels of cTnI and CK-MB was measured by chemiluminescence immunoassay using Maglumi 800 fully automated chemiluminescence immunoassay analyser via Shenzhen New Industries Biomedical Engineering Co, Ltd protocol.

\section{Data analysis}

Finally, we analysed the result and compared the pre-training test to after 1 week tests as well as between group results. Analysis of the data obtained from the study inferential statistics was employed. To evaluate whether or not the data are normally distributed the Kolmogorov-Smirnov and Shapiro-Wilk ${ }^{28}{ }^{29}$ normality test was applied. In case of normal distribution, Student's paired t-test was used for an inter group (pre-post) comparison and to compare the effects of high and moderate intensity endurance training on physical performances and serum cardiac stress markers of athletes; one way between group analysis of variance was carried out using 
Table 1 General characteristics of participants

\begin{tabular}{|c|c|c|c|}
\hline & $\begin{array}{l}\text { Moderate } \\
\text { intensity training } \\
\text { group }\end{array}$ & $\begin{array}{l}\text { High intensity } \\
\text { training group }\end{array}$ & ANOVA \\
\hline Parameters & $M \pm S D$ & $M \pm S D$ & $P$ value \\
\hline Age & $19.27 \pm 1.28$ & $19.47 \pm 1.30$ & 0.67 \\
\hline Height & $1.67 \pm 0.06$ & $1.67 \pm 0.07$ & 0.84 \\
\hline Body mass & $53.87 \pm 4.76$ & $53.96 \pm 5.67$ & 0.964 \\
\hline $\mathrm{BMI}$ & $19.39 \pm 1.49$ & $19.31 \pm 1.41$ & 0.881 \\
\hline
\end{tabular}

ANOVA, analysis of variance; $B M I$, body mass index; $M \pm S D$, mean and standard deviation; $P$, probability.

IBM-SPSS V.20. Each result of the data is expressed as mean \pm SD. All statistical inferences were seen at $(p \leq 0.05)$ level of significance.

\section{RESULTS}

No between-group differences (age, height, body mass and body mass index) existed at baseline, so the groups were well matched at entry (table 1 ).

Markers of cardiorespiratory and cardiometabolic response of pre and post 1 week endurance exercise training are presented in (table 2). Both intensity programme had no significant effect on markers of cardiorespiratory response within and between training groups $(p>0.05)$. Mean values for cardiometabolic markers LDH $(p=0.001)$, cTnI $(p=0.001)$ and CK-MB $(p=0.001)$ significantly increased at the end of 1 week within the group in both training intensity zones $(p \leq 0.05)$. In the comparative analysis between training groups, there was no significant difference in markers of cardiometabolic response $(p>0.05)$.

\section{DISCUSSION}

This study compared the effects of short-different intensity endurance training on markers for cardiorespiratory and cardiometabolic responses in young moderately trained athletes. Our main finding observed no major difference in markers for cardiorespiratory and cardiometabolic markers between training groups. However, a significant difference was observed in markers for cardiometabolic and but not cardiorespiratory within training groups.

Consistent with our finding, previous studies reported no difference in markers for cardiorespiratory responses between and within intensity training groups. ${ }^{14-16}$ Furthermore, a study by Gormley $e t a \hat{l}^{30}$ administered endurance training under various intensities and reported no significant difference in markers for cardiorespiratory responses, between groups. Indeed, a meta-analysis, that coded training status and similar training intensities in sedentary subjects, reported that every training intensity from below lactate threshold to above $4 \mathrm{mmol} \cdot \mathrm{L}^{-1}$ blood lactate concentration improved performance, with no significant difference between the intensities. Thus, the reason for no observed differences could be due to administering endurance training either at or below lactate threshold that equally evoke a similar physiological response in amateur athletes. ${ }^{31}{ }^{32}$ Therefore, if the main goal of endurance exercise is to improve markers for cardiorespiratory responses for amateur athletes, it would be better done under moderate intensity training similar to $60 \%$ to $70 \%$ HRmax. Considering that younger athletes have been reported to possess reduced arterial stiffness and might respond to short-term endurance exercise. $^{33}$

Studies by Peters et $a \ell^{34}$ and Baker et $a l^{35}$ reported that moderate and high intensity endurance training corresponds to the designed intensities in our modified

Table 2 A comparison of cardiorespiratory and cardiometabolic responses between and within the groups

\begin{tabular}{|c|c|c|c|c|c|c|c|c|}
\hline \multirow[b]{3}{*}{ Variables } & \multicolumn{2}{|c|}{ Moderate intensity group } & \multirow[b]{3}{*}{$P$ value } & \multicolumn{2}{|c|}{ High intensity group } & \multicolumn{2}{|c|}{ between groups } & \multirow[b]{3}{*}{$P$ value } \\
\hline & Pre-test & Post-test & & Pre-test & Post-test & $P$ value & ANOVA & \\
\hline & $\mathrm{M} \pm \mathrm{SD}$ & $\mathrm{M} \pm \mathrm{SD}$ & & $M \pm S D$ & $\mathrm{M} \pm \mathrm{SD}$ & & $\mathbf{F}$ & \\
\hline \multicolumn{9}{|c|}{ Cardiorespiratory markers } \\
\hline Systolic BP & $120.5 \pm 8.3$ & $119.4 \pm 10.3$ & 0.762 & $113.5 \pm 5.5$ & $114.5 \pm 6.5$ & 0.644 & 7.449 & 0.01 \\
\hline Diastolic BP & $74.1 \pm 8.5$ & $77.5 \pm 9.80$ & 0.242 & $73.5 \pm 8.7$ & $74.1 \pm 6.9$ & 0.828 & 0.29 & 0.866 \\
\hline MAP & $89.5 \pm 7.1$ & $91.6 \pm 9.7$ & 0.454 & $86.8 \pm 6.5$ & $87.3 \pm 4.8$ & 0.759 & 2.275 & 0.143 \\
\hline RHR & $75.9 \pm 8.3$ & $71.7 \pm 0.6$ & 0.250 & $71.2 \pm 8.8$ & $71.7 \pm 7.0$ & 0.872 & 2.235 & 0.146 \\
\hline $\mathrm{VO}_{2} \max$ & $60.7 \pm 4.5$ & $62.2 \pm 4.7$ & 0.345 & $60.4 \pm 4.5$ & $60.8 \pm 4.2$ & 0.082 & 0.46 & 0.831 \\
\hline \multicolumn{9}{|c|}{ Cardiometabolic markers } \\
\hline LDH & $136 \pm 38.4$ & $245.8 \pm 102.1$ & 0.002 & $178.9 \pm 52$ & $297.5 \pm 10$ & 0.001 & 1.961 & 0.172 \\
\hline cTnl & $0.10 \pm 0.11$ & $0.44 \pm 0.22$ & 0.0001 & $0.12 \pm 0.12$ & $0.59 \pm 0.32$ & 0.0001 & 2.482 & 0.126 \\
\hline CK-MB & $1.12 \pm 0.97$ & $3.39 \pm 10$ & 0.0002 & $1.71 \pm 1.4$ & $4.1 \pm 1.6$ & 0.00001 & 1.764 & 0.195 \\
\hline
\end{tabular}

ANOVA, analysis of variance; BP, blood pressure; CK-MB, creatine kinase myocardial band; cTnl, cardiac troponin I; LDH, lactate dehydrogenase; MAP, mean arterial pressure; $\mathrm{M} \pm \mathrm{SD}$, mean and standard deviation; RHR, resting heart rate; $\mathrm{VO}_{2}$ max, maximum oxygen consumption. 
protocol does not lead to elevations in serum markers of cardiometabolic responses within the groups in amateur athletes. However, elevation in serum markers of cardiometabolic responses within groups has been reported $^{10} 13$ 35-37 and our findings are in agreement. Besides, a study by Bauer $e$ t $a l^{88}$ and Callegari $e t a l^{39}$ also reported an elevation in serum markers of cardiometabolic responses in elite athletes. Interestingly, exercise is possibly the only documented cause of increases serum markers of cardiometabolic responses such as cardiac troponin that is not associated with adverse clinical outcome. This has led to the hypothesis that an elevation in serum markers of cardiometabolic responses is part of a normal physiological response to exercise and does not represent irreversible cell death. It is important to highlight that, the elevation in serum markers of cardiometabolic responses is often associated with younger age and might reflect poor adaptation to endurance training. ${ }^{35}$ Since, poor adaptation to endurance training in young athletes has been related to increased muscle and cartilage inflammation ${ }^{3}$ that may result to increased serum cardiometabolic markers. Consequently, our finding and others ${ }^{39}$ did not observe differences in serum markers of cardiometabolic response between short-term different intensity endurance training groups. Whether elevation of serum markers cardiometabolic responses of young athletes might indicate poor or better adaptation to endurance training needs further investigation.

Exercise intensity at the boundary of moderate-to-high intensity ${ }^{40}$ can be maintained for a prolonged period of time and is therefore very attractive to untrained individuals explained as decreased cardiac markers as maximum oxygen consumption increased after training adaptation. ${ }^{41}$ Our findings imply that training intensity zones $60 \%$ to $70 \%$ HRmax or $70 \%$ to $80 \%$ HRmax, may induce similar effects on serum markers for cardiometabolic and cardiorespiratory in amateur athletes. However, different training duration is the limitation of this study. It is likely that in young athletes these events are a normal, potentially adaptive, process associated with the work of prolonged exercise. Further study is required, whether these changes are associated with tissue damage and what the long-term consequences of such maladaptation might be.

\section{CONCLUSION}

One week $60 \%$ to $70 \%$ HRmax and $70 \%$ to $80 \%$ HRmax intensity zones induces similar acute effects on markers for cardiometabolic and cardiorespiratory responses in amateur athletes between groups and significant elevations of cardiometabolic markers within the groups.

Acknowledgements The Authors thank Mekelle University and Mizan-Tepi University for their support.

Contributors All authors were involved in the design and concept of the study. MG; involved in the consulting of the design and method of the study as well as in writing the manuscript, SM; was involved in consulting the data gathering time and tools, DM; was involved in designing theoretical frame work of the study and GT; involved from the conception to experiment and writing the manuscript.

Funding The authors have not declared a specific grant for this research from any funding agency in the public, commercial or not-for-profit sectors.

Competing interests None declared.

Patient consent for publication Not required.

Ethics approval Ethical approval was obtained from Institutional Research Ethics Review Committee of Mekelle University (Ethiopia): With the reference number ERC 1079/2017. Written consent was delivered and signed by the participants and they were informed about the objectives of the study.

Provenance and peer review Not commissioned; externally peer reviewed.

Data availability statement All data relevant to the study are included in the article or uploaded as supplementary information.

Open access This is an open access article distributed in accordance with the Creative Commons Attribution 4.0 Unported (CC BY 4.0) license, which permits others to copy, redistribute, remix, transform and build upon this work for any purpose, provided the original work is properly cited, a link to the licence is given, and indication of whether changes were made. See: https://creativecommons.org/ licenses/by/4.0/.

\section{REFERENCES}

1. Black KE, Skidmore PML, Brown RC. Energy intakes of ultraendurance cyclists during competition, an observational study. Int J Sport Nutr Exerc Metab 2012;22:19-23.

2. Fallon KE, Sivyer G, Sivyer K, et al. The biochemistry of runners in a 1600 Km ultramarathon. Br J Sports Med 1999;33:264-9.

3. Kim HJ, Lee YH, Kim CK. Biomarkers of muscle and cartilage damage and inflammation during a $200 \mathrm{Km}$ run. Eur J Appl Physiol 2007;99:443-7.

4. Del Coso J, Fernández de Velasco D, Fernández D, Abian-Vicen J, et al. Running PACE decrease during a marathon is positively related to blood markers of muscle damage. PLoS One 2013;8:e57602.

5. Seiler S, Stephen $\mathrm{S}$. What is best practice for training intensity and duration distribution in endurance athletes? Int J Sports Physiol Perform 2010;5:276-91.

6. Seiler S, Intervals TE. Thresholds, and long slow distance: the role of intensity and duration in endurance training. Sportscience 2009;13:32-53.

7. Perry CGR, Heigenhauser GJF, Bonen A, et al. High-Intensity aerobic interval training increases fat and carbohydrate metabolic capacities in human skeletal muscle. Appl Physiol Nutr Metab 2008;33:1112-23.

8. Trapp EG, Chisholm DJ, Freund JBS. The effects of high intensity intermittent exercise training on fat loss and fasting insulin levels of young women. Int J Obes 2008;32:684-91.

9. Serrano-Ostáriz E, Terreros-Blanco JL, Legaz-Arrese A, et al. The impact of exercise duration and intensity on the release of cardiac biomarkers. Scand J Med Sci Sports 2011;21:244-9. 2.

10. Serrano-Ostáriz E, Terreros-Blanco JL, Terreros-Blanco JL, et al. Cardiac biomarkers and exercise duration and intensity during a cycle-touring event. Clin J Sport Med 2009;19:293-9.

11. Bird SR, Linden M, Hawley JA. Acute changes to biomarkers as a consequence of prolonged strenuous running. Ann Clin Biochem 2014;51:137-50.

12. Park $\mathrm{C}-\mathrm{H}$, Kim K-B, Han J, et al. Cardiac damage biomarkers following a Triathlon in elite and Non-elite triathletes. Korean $J$ Physiol Pharmacol 2014;18:419-23.

13. Peake JM, Suzuki K, Wilson G, et al. Plasma cytokines, and markers of neutrophil activation. Med Sci Sport Exerc 2005;37:737-45.

14. Daussin FN, Zoll J, Dufour SP, et al. Effect of interval versus continuous training on cardiorespiratory and mitochondrial functions: relationship to aerobic performance improvements in sedentary subjects. Am J Physiol Regul Integr Comp Physiol 2008;295:R26 4-R272.

15. Gorostiaga EM, Walter CB, Foster C, et al. Uniqueness of interval and continuous training at the same maintained exercise intensity. Eur J Appl Physiol Occup Physiol 1991;63:101-7.

16. Helgerud J, Hoydal K, Wang E, et al. Aerobic High-Intensity Intervals Improve V??O2max More Than Moderate Training. Medicine \& Science in Sports \& Exercise 2007;39:665-71.

17. Febiger $L$ and. Guidelines for exercise testing and priscription. 4th ed. ACSM, 1991: 73-4.

18. Haile Z. Assessment of climate change impact on the net Basin supply of lake Tana water balance. International Institute for 
geo- information science and earth observation ENSCHEDE. The Netherlands, 2009.

19. Sivaraman P. Effect of eight weeks aerobic exercises on physical and physiological variables among College men. IJPEFS 2014;3:113-7.

20. Chow S-C. Jun Shao and, Wang H. sample size calculations in clinical research. second edi. Chapman \& Hall/CRC Taylor \& Francis Group 2008.

21. Shephard RJ. Physical activity participation clearance and prescription. Heal Ftness J Canada 2014;7.

22. Barnes MJ, Barnes MJ. Alcohol: impact on sports performance and recovery in male athletes. Sports Med 2014;44:909-19.

23. Lee C-L, Chang W-D. The effects of cigarette smoking on aerobic and anaerobic capacity and heart rate variability among female university students. Int J Womens Health 2013;5:667-79.

24. Marquaria R, Aghema PRE. Determination of maximal oxygen consumption in man. J Appl Physiol 1965;20:1070-3.

25. McArdle WD KF\& KV. Exercise physiology: energy, nutrition and human performance. 7th edn. Baltimore: Williams \& Williams, 2010.

26. Legaz-Arrese A, López-Laval I, George K, et al. Impact of an endurance training program on exercise-induced cardiac biomarker release. Am J Physiol Heart Circ Physiol 2015;308:H913-H920.

27. Tian $\mathrm{Y}, \mathrm{Nie} \mathrm{J}$, Huang $\mathrm{C}$, et al. The kinetics of highly sensitive cardiac troponin $T$ release after prolonged treadmill exercise in adolescent and adult athletes. J Appl Physiol 2012;113:418-25.

28. Razali \& Wah. Power comparisons of shapiro-Wilk, KolmogoroveSmirnove,Lilliefors and Andersin-darling tests. J Stat Model Anal 2011;5:20-33.

29. Shapiro SS, Wilk MB. An analysis of variance test for normality (complete samples). Biometrika 1965;52:591-611. 3/4.

30. Gormley SE, Swain DP, High R, et al. Effect of Intensity of Aerobic Training on V??O2max. Medicine \& Science in Sports \& Exercise 2008; $40: 1336-43$

31. Stasiulis A, Stasiulis A, Romutis Ančlauskas JJ, et al. The effects of training intensity on blood lactate breakpoints in runners. J Ournal Hum Kinet 2000;3.
32. Londeree BENR, Londeree B. Effect of training on lactate/ventilatory thresholds: a meta-analysis. Medicine \&amp Science in Sports \&amp Exercise 1997;29:837-43.

33. Tanaka M, Sugawara M, Ogasawara Y, et al. Intermittent, moderateintensity aerobic exercise for only eight weeks reduces arterial stiffness: evaluation by measurement of stiffness parameter and pressure-strain elastic modulus by use of ultrasonic echo tracking. $J$ Med Ultrason 2013;40:119-24.

34. Peters M ECD, Anton $\mathrm{H}$ de Waard NRS. Anton $\mathrm{H}$ de Waard NRS. low markers of muscle damage and inflammation following a 3-day TRAIL run. SAJSM 2012;24.

35. Baker P, Davies SL, Larkin J, et al. Changes to the cardiac biomarkers of non-elite athletes completing the 2009 London marathon. Emerg Med J 2014;31:374-9.

36. Eijsvogels $\mathrm{TMH}$, Hoogerwerf MD, Oudegeest-Sander MH, et al. The impact of exercise intensity on cardiac troponin I release. Int $J$ Cardiol 2014;171:e3-4.

37. Serrano-Ostáriz E, Terreros-Blanco JL, Legaz-Arrese A, et al. The impact of exercise duration and intensity on the release of cardiac biomarkers. Scand J Med Sci Sports 2011;21:244-9.

38. Bauer P, Zeißler S, Walscheid R, et al. D A. changes of cardiac biomarkers after high-intensity exercise in male and female elite athletes of dragon Boating. J Sport Sci 2016;4:1-8.

39. Callegari GA, Novaes JS, Neto GR, et al. Creatine kinase and lactate dehydrogenase responses after different resistance and aerobic exercise protocols. J Hum Kinet 2017;58:65-72.

40. Beneke R. Anaerobic threshold, individual anaerobic threshold, and maximal lactate steady state in rowing. Medicine \& Science in Sports \& Exercise 1995;27:863???867-7.

41. Tesema G, George M, Mondal S, et al. Serum cardiac markers are inversely associated with $\mathrm{VO}_{2}$ max of amateur athletes in response to endurance training adaptations. BMJ Open Sport Exerc Med $2019 ; 5$. 